\title{
Scaling of anisotropic droplet shapes on chemically stripe-patterned surfaces
}

\author{
O. Bliznyuk, ${ }^{1}$ E. Vereshchagina, ${ }^{2}$ E. Stefan Kooij,${ }^{1, *}$ and Bene Poelsema ${ }^{1}$ \\ ${ }^{1}$ Solid State Physics, IMPACT, University of Twente, P.O. Box 217, NL-7500AE Enschede, The Netherlands \\ ${ }^{2}$ Mesoscale Chemical Systems, MESA + Institute, University of Twente, P.O. Box 217, NL-7500AE Enschede, The Netherlands
}

(Received 14 October 2008; revised manuscript received 3 March 2009; published 3 April 2009)

\begin{abstract}
We present an experimental study of the tunable anisotropic wetting behavior of chemically patterned anisotropic surfaces. Asymmetric glycerol droplet shapes, arising from patterns of alternating hydrophilic (pristine $\mathrm{SiO}_{2}$ ) and hydrophobic (fluoroalkylsilane self-assembled monolayers) stripes with dimensions in the low-micrometer range, are investigated in relation to stripe widths. Owing to the well-defined small droplet volume, the equilibrium shape as well as the observed contact angles exhibit unique scaling behavior. Only the relative width of hydrophilic and hydrophobic stripes proves to be a relevant parameter. Our results on morphologically flat, chemically patterned surfaces show similarities with those of experiments on topographically corrugated substrates. They are discussed in terms of the energetics at the liquid-solid interface.
\end{abstract}

DOI: 10.1103/PhysRevE.79.041601

PACS number(s): 68.03.Cd, 68.08.Bc

\section{INTRODUCTION}

Controlling surface wettability is attracting significant scientific attention in many research areas, including fluid physics, materials science, and interface physics. Applications of "smart" surfaces with artificially designed wetting properties range, for example, from micro- or nanofluidic devices to car windows. Numerous theoretical and experimental studies have been conducted on chemically heterogeneous [1-7] and topographically structured surfaces [8-12].

Following the identification of surface roughness as the origin of the "lotus effect" [13] and the race to pursue artificial superhydrophobic surfaces, most research has concentrated on investigating the behavior of liquids on isotropic, topographically patterned surfaces [14]. Nevertheless, anisotropic surfaces can provide an important insight into the effect of dynamics on the final shape of the sessile droplet, therewith enabling one to gain better understanding of the role of surface geometry $[15,16]$. One of the first and nowadays still frequently studied anisotropic structured periodic surfaces consists of parallel grooves $[17,18]$. The popularity of these surfaces originates from the relative ease to manufacture them and simple modeling with only few parameters [19].

The investigation of similar anisotropic two-dimensional systems on morphologically flat substrates using chemical surface modification was hindered by the difficulty to reproducibly obtain sufficiently small features. Recent advances in the field of self-assembled monolayers (SAMs) and their application in surface modification, combined with progress in lithographic patterning tools, enable reproducible manufacture of well-defined patterns to be used in wettability studies [20]. Here we present an experimental study specifically focused on chemically heterogeneous surfaces and their influence on the final shape of sessile drops. The well-defined small droplet volume used in our experiments allows experimental identification of anisotropic wetting properties in terms of scaling behavior.

\footnotetext{
*e.s.kooij@tnw.utwente.nl
}

\section{EXPERIMENTAL DETAILS}

The surface patterns employed in our investigation consist of alternating hydrophobic and hydrophilic stripes (fluoroalkylsilane $\mathrm{SAMs}$ and bare $\mathrm{SiO}_{2}$ surface, respectively), giving rise to anisotropic wetting properties as schematically shown in Fig. 1. Using standard cleanroom facilities, silicon wafers with a thin layer of natural oxide are coated with positive photoresist, enabling pattern creation via optical lithography and providing surface protection during vapor

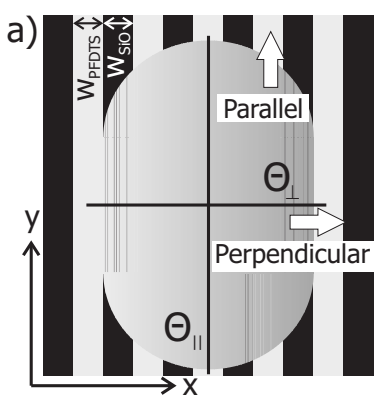

b)

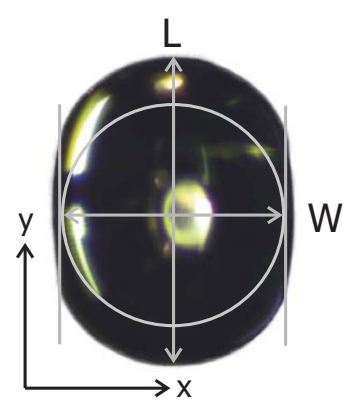

c)
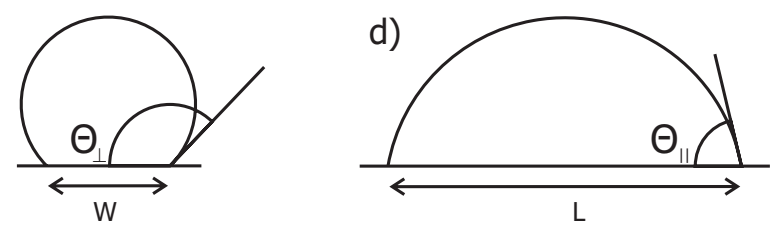

FIG. 1. (Color online) Quantifying anisotropic drops: (a) schematic top-view representation and (b) photograph of an asymmetric (glycerol) droplet on a chemically patterned surface consisting of alternating $\mathrm{SiO}_{2}$ (hydrophilic) and $1 \mathrm{H}, 1 \mathrm{H}, 2 \mathrm{H}, 2 \mathrm{H}$ perfluorodecyltrichlorosilane (PFDTS) (hydrophobic) stripes. The width of the stripes was varied in the range of 2-20 $\mu \mathrm{m}$. The relative width of the stripes leads to a variation from a predominantly hydrophilic surface (wider $\mathrm{SiO}_{2}$ ) to mostly hydrophobic substrates (wider PFDTS). Two spreading regimes perpendicular and parallel to the stripes ( $x$ and $y$ directions) lead to different macroscopic contact angles $\Theta_{\perp}$ and $\Theta_{\|}$and corresponding width $W$ and length $L$, as schematically depicted in (c) and (d), respectively. Note that the actual number of lines underneath the droplet is much larger than shown in (a). 


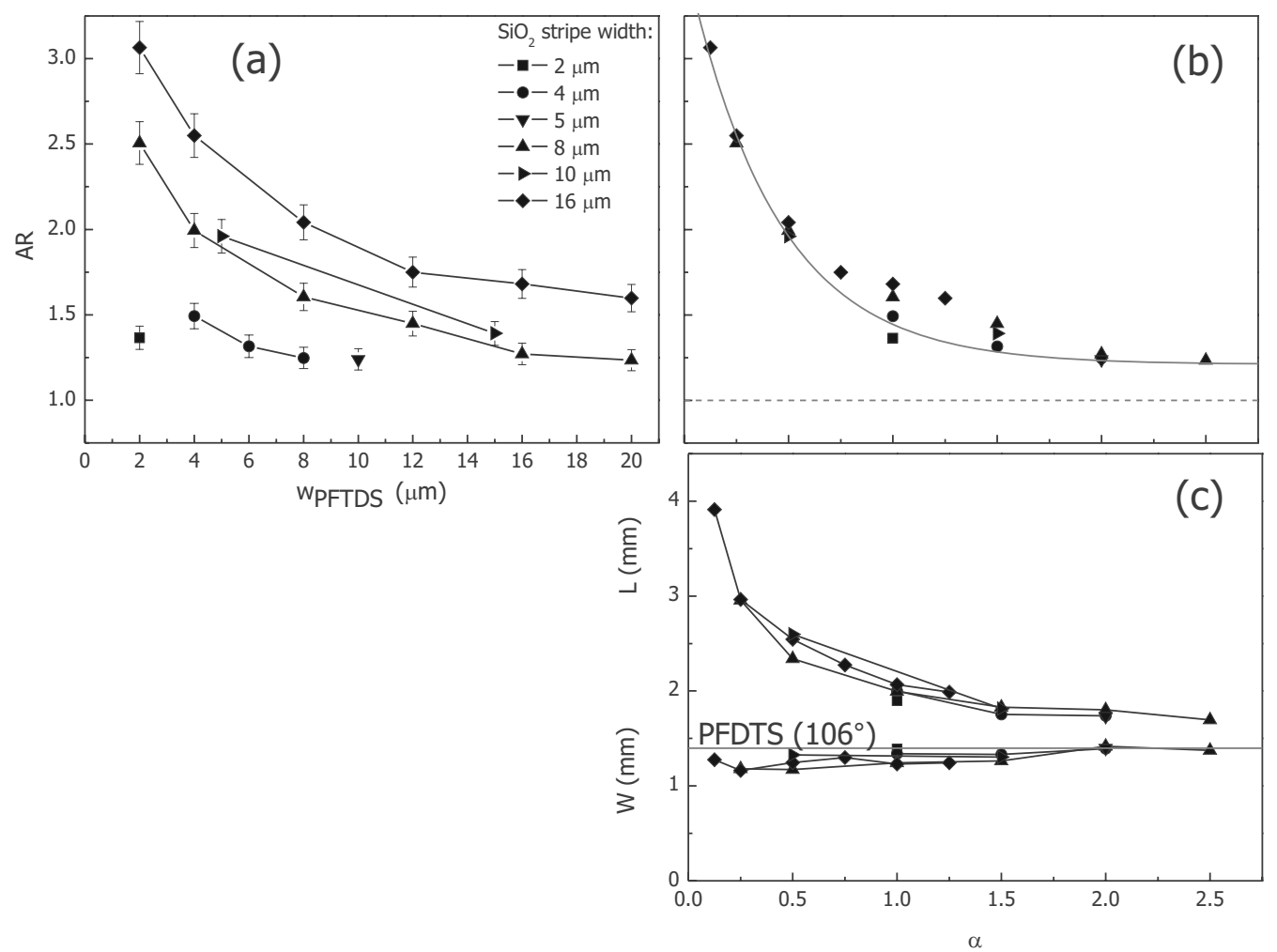

FIG. 2. Experimentally determined droplet shapes in relation to the underlying chemical pattern. (a) Aspect ratio as a function of PFDTS stripe width, for various $\mathrm{SiO}_{2}$ stripe widths. (b) Aspect ratio as a function of the relative widths of PFDTS and $\mathrm{SiO}_{2}$ stripes exhibits scaling behavior; the solid line is a guide for the eyes. (c) Length and width of the fixed-volume $(1 \mu \mathrm{l})$ droplets reveal that the variation in the aspect ratio is primarily due to length changes. The horizontal gray line indicates the diameter of spherical cap-shaped drops of fixed volume on unpatterned PFDTS for the contact angle as indicated.

deposition of PFDTS (ABCR, Germany). After SAM formation the photoresist is washed off, leaving a chemically patterned surface. Formation of PFDTS SAM is confirmed by atomic force microscopy (AFM). The AFM pictures show well-defined borders between a PFDTS SAM and a $\mathrm{SiO}_{2}$ region; the PFDTS thickness is measured to be $0.8 \mathrm{~nm}$.

Droplet deposition and characterization, including measurement of contact angles (CAs), is done using an OCA 15+ apparatus (Dataphysics, Germany). The equipment enables determination of CAs with an accuracy below $0.5^{\circ}$; in all cases we performed multiple measurements. The experimental variation in CAs on identical samples was less than $2^{\circ}$. An additional top-view camera is mounted to assess the inplane droplet shape.

Droplets are created using a computer-controlled syringe. Due to surface tension, the droplets are suspended below the syringe needle. Deposition of the droplet is achieved by very slowly lowering the suspended droplet onto the surface. As soon as the droplet is in contact with the surface, wettinginduced spreading leads to detachment from the needle, after which the final shape is reached within a minute. For all droplets, the volume is fixed to $1 \mu \mathrm{l}$; the variation in droplet volume was less than 5\%. The liquid used is glycerol (Glycerol ReagentPlus, Sigma, USA). The surface tensions of water and glycerol are nearly the same, giving rise to very similar behavior of both liquids. However, with glycerol, evaporation is considerably less, therewith enabling timeconsuming experiments. As droplet dimensions are in the millimeter range, our surfaces are considered to be flat, but chemically heterogeneous. Linewidths are varied in the range of 2-20 $\mu \mathrm{m}$; droplets span 700 to 80 lines. For every data point, measurements were performed on two or three different substrates (with identical patterns). For each substrate, at least two droplets were deposited and characterized. For each of these droplets (i.e., at least four measurements), the aspect ratio is determined, values of which are subsequently averaged.

\section{DROPLET ANISOTROPY}

The unidirectional chemical pattern on our surfaces induces anisotropic wetting properties, which in turn lead to different spreading behavior in orthogonal directions. This gives rise to an equilibrium situation in which the droplet shape deviates from spherical, such as that shown in Fig. 1. In fact, the top view of the droplets reveals that the shape can be approximated by a cylinder with two spherical caps. To quantify the distortion from a spherical shape, we introduce the aspect ratio $\mathrm{AR}=L / W$, where $L$ represents the size parallel to the stripes and $W$ is defined as the width at the solidliquid interface, as shown in Fig. 1(c). The droplet shape strongly depends on the width of the hydrophilic and hydrophobic lines, as depicted in Fig. 2(a). For fixed $\mathrm{SiO}_{2}$ stripe widths (connected symbols), the AR increases markedly with decreasing PFDTS stripe widths. This is most pronounced for the largest $\mathrm{SiO}_{2}$ stripe widths. For smaller $\mathrm{SiO}_{2}$ stripe 
width, the variation in the AR is markedly smaller.

To investigate whether the shape distortion is governed by a universal mechanism, we plot the AR as a function of the relative width of PFDTS to $\mathrm{SiO}_{2}$ stripes. The hydrophobicto-hydrophilic ratio is defined by a dimensionless parameter $\alpha$ given by

$$
\alpha=\frac{w_{\mathrm{PFDTS}}}{w_{\mathrm{SiO}_{2}}},
$$

where $w_{\mathrm{PFDTS}}$ and $w_{\mathrm{SiO}_{2}}$ are the hydrophobic and hydrophilic stripe widths, respectively. In our experiments the ratio $\alpha$ varies between 0.125 and 6 , where $\alpha<1$ and $\alpha>1$ correspond to more hydrophilic and hydrophobic surfaces, respectively. For $\alpha=1$ the hydrophobic and hydrophilic areas are equal.

Figure 2(b) reveals that the AR plotted as a function of $\alpha$ indeed leads to a single curve for all patterns. Apparently, the relative hydrophobicity, i.e., the ratio $\alpha$, is the predominant universal parameter defining the droplet shape. We stress that this only holds as long as the droplet spans many lines or the pattern dimensions remain in the micrometer range [21], i.e., variations in the liquid-vapor interface especially near the triple line can be neglected.

Clearly observable in Fig. 2(b) is the pronounced decrease in the AR with increasing $\alpha$ for more hydrophobic surfaces. For $\alpha>2.5$ (not shown in Fig. 2) the droplets appear almost spherical. Moreover, the corresponding CAs larger than 90 hinder accurate determination of the triple line that marks the border of the droplet.

Most surprising is the asymmetry observed in Fig. 2. For $\alpha=2$ the exposed $\mathrm{SiO}_{2}$ area amounts to $1 / 3$ of the total area, giving rise to very limited anisotropy. On the other hand, for $\alpha=0.5$, at which the PFDTS-coated area amounts to $33 \%$, this leads to highly asymmetric droplet shapes. Further reduction in the relative PFDTS-coated area leads to even more elongated droplets, with $\mathrm{AR} \geq 3$. Note that for $\alpha=0$ (pure $\mathrm{SiO}_{2}$ surface), obviously, we observe perfectly spherical drop shapes with $\mathrm{AR}=1$.

Furthermore, in Fig. 2(c) the length $L$ and width $W$ are plotted as functions of $\alpha$. The length $L$ varies from 1.5 to 4.0 $\mathrm{mm}$, where the latter value (for $\alpha=0.125$ ) corresponds to the calculated droplet diameter on clean $\mathrm{SiO}_{2}$, while $W$ values are obtained within $0.3 \mathrm{~mm}$ of the theoretical value of 1.39 $\mathrm{mm}$ for clean PFDTS SAMs. This plot reveals that the elongation parallel to the stripes dominates the aspect ratio of the droplets, while the width variation is much smaller. Moreover, both $\mathrm{AR}$ and $L$ exhibit similar scaling behavior.

The horizontal line in Fig. 2(c) corresponds to the theoretical widths of droplets with identical volume on pure PFDTS surfaces (no anisotropy), considering an average value of $\mathrm{CA}=106^{\circ}$ as observed in our experiments. For low $\alpha$ values corresponding to highly anisotropic droplet shapes, the width is lower. For $\alpha \geq 2$ the observed widths are approximately equal to that of droplets on pure PFDTS-coated surfaces. Scattering of the width values can be explained by "pinning," i.e., hindered spreading of the triple line in the $x$ direction at the pattern edges. The triple line experiences a finite free-energy barrier for every hydrophobic PFDTS stripe it will attempt to bridge [22-24].

\section{ANISOTROPIC WETTABILITY}

The wetting characteristics of our chemically heterogeneous surfaces are investigated by measuring the profiles of sessile droplets. CAs are measured perpendicular $\left(\Theta_{\perp}\right)$ and parallel $\left(\Theta_{\|}\right)$to the stripes, as schematically shown in Fig. 1 [25]. These angles reflect the limiting values, belonging to the two different spreading regimes of a liquid on the patterned surface. The variation in the CAs measured as a function of the horizontal viewing angle, i.e., around the contour of a droplet on a $50 \%$ hydrophilic-50\% hydrophobic surface is illustrated in Fig. 3(a). When viewing the droplets "from the side" (at small or large view angles; a view angle of $0^{\circ}$ or $180^{\circ}$ is perpendicular to the stripes, as indicated by the white arrows), the CA $\Theta_{\|}$parallel to the stripes is observed. For a view angle of $90^{\circ}$ we only "see" the perpendicular CA $\Theta_{\perp}$. Upon rotating the view angle from $0^{\circ}$ or $180^{\circ}$ toward $90^{\circ}$, the CAs remain near $80^{\circ}$, until at $90^{\circ} \pm 30^{\circ}$ the CAs rapidly increase. The constant low values of $\Theta_{\|}$in the view angle ranges of $0^{\circ}-50^{\circ}$ and $130^{\circ}-180^{\circ}$ reflect the fact that along the circular parts of the droplet contour, the CA remains unchanged. Only when the view angle of the droplet profile is almost parallel to the stripe pattern, is a sharp increase observed. In fact, the actual increase in the CA is much "sharper," but the fact that the entire droplet (and not only a cross section) is viewed leads to a more gradually observed change.

In the direction parallel to the stripes, the droplet contour "feels" the rapidly varying hydrophilic/hydrophobic nature of the surface. As such, the CA value is expected to be mostly defined by the chemical composition of the surface underneath the droplet. In fact, in contrast to $\Theta_{\perp}, \Theta_{\|}$is not a real CA, but an effective (macroscopic) CA, which is obtained by averaging the (microscopic) equilibrium CAs exhibited by the $\mathrm{SiO}_{2}$ and the PFDTS stripes $[21,26]$. In this assumption, only the relative hydrophobicity, i.e., the value $\alpha$ in Eq. (1), should influence the observed CA. Thus scaling behavior is expected, similar to that in Fig. 2 . The results in Fig. 3(b) confirm that indeed the CAs scale onto a single curve, increasing from approximately $40^{\circ}$ at low $\alpha$ values toward $100^{\circ}$ at large $\alpha$ values.

Considering the droplet dimensions to be at least 1 order of magnitude larger than the pattern stripe widths, the droplet resides on an effectively (chemically) heterogeneous surface. The CA is defined by the areal contribution of each species and we assume that the observed $\Theta_{\|}$can be modeled by Cassie's law [27,28], which for a binary composite surface is given by

$$
\cos \Theta_{\|}=f_{1} \cos \Theta_{1}+f_{2} \cos \Theta_{2},
$$

where $f_{1}$ and $f_{2}$ correspond to area fractions that exhibit CAs of $\Theta_{1}$ and $\Theta_{2}$, respectively. Considering the equilibrium CAs $\Theta_{\text {PFDTS }}$ and $\Theta_{\mathrm{SiO}_{2}}$ on $100 \%$ PFDTS and $100 \% \mathrm{SiO}_{2}$ surfaces, respectively, and inserting the relative area fractions $\alpha /(1$ $+\alpha)$ of PFDTS and $1 /(1+\alpha)$ for $\mathrm{SiO}_{2}$, rewriting Eq. (2) yields a relation between the parallel CA $\Theta_{\|}$and the scaling parameter $\alpha$, 

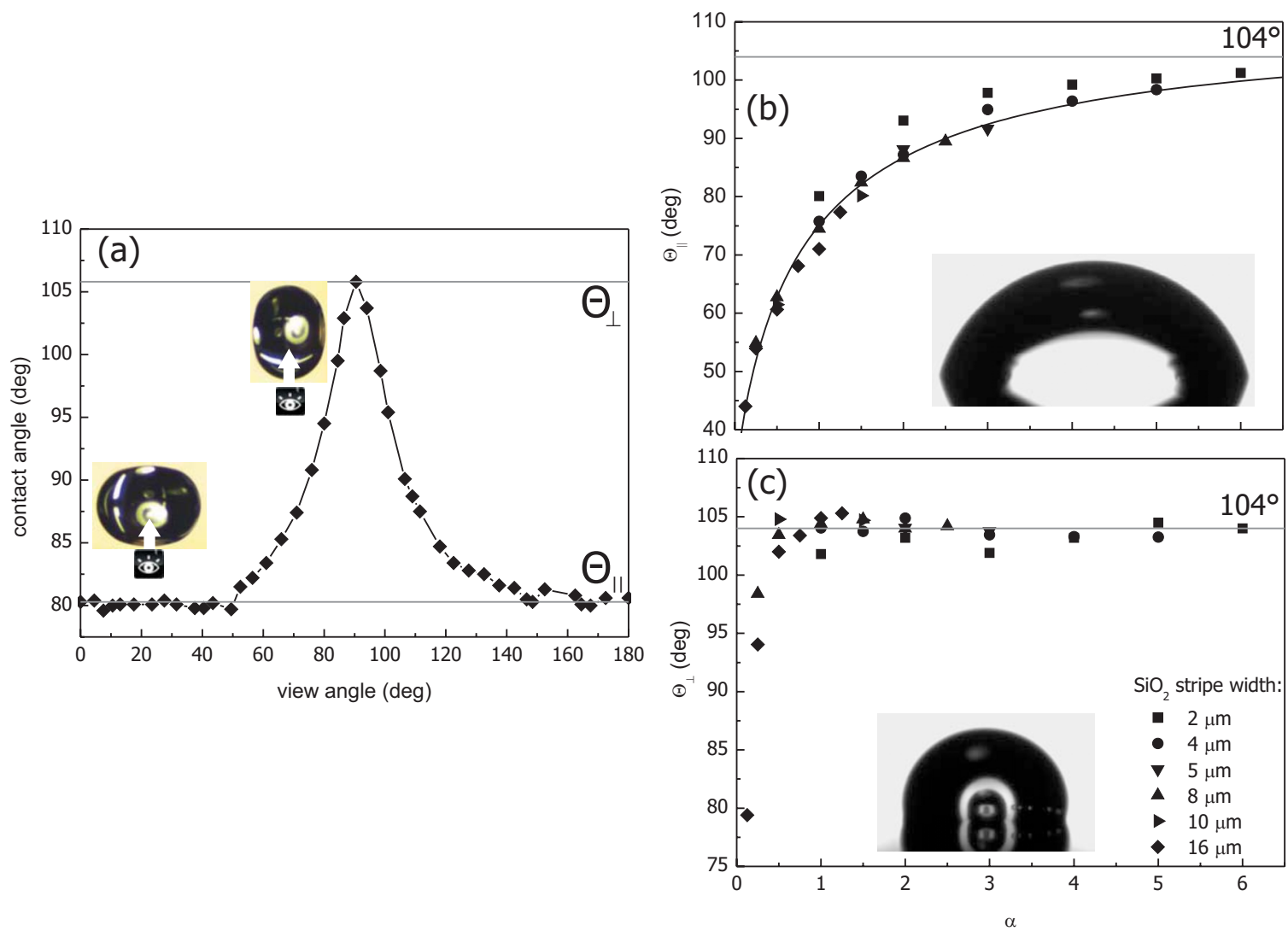

FIG. 3. (Color online) (a) Contact angles measured along the contour of a sessile droplet with $\mathrm{AR} \approx 1.5$ on $8 \mu \mathrm{m} \mathrm{SiO} \mathrm{S}_{2}-8 \mu \mathrm{m}$ PFDTS pattern; the insets depict the limiting viewing angles, indicated by the white arrow. [(b) and (c)] Contact angles parallel $\left(\Theta_{\|}\right)$and perpendicular $\left(\Theta_{\perp}\right)$ to the stripe pattern as functions of $\alpha$. Similarly as with the results in Fig. 2, the data scale to a single line that is described by Cassie's law (solid line).

$$
\Theta_{\|}=\arccos \left[\frac{\alpha \cos \left(\Theta_{\mathrm{PFDTS}}\right)+\cos \left(\Theta_{\mathrm{SiO}_{2}}\right)}{1+\alpha}\right] .
$$

The scaled data in Fig. 3(b) were used to fit Eq. (3), using the equilibrium CAs on pristine $\mathrm{SiO}_{2}$ and PFDTS as fitting parameters. The best fit for the experimental data was obtained for $\Theta_{\mathrm{PFDTS}}=110^{\circ}$ and $\Theta_{\mathrm{SiO}_{2}}=31^{\circ}$ and is shown by the solid line in Fig. 3(b). Despite the slight deviation from CAs measured on actual pristine surfaces $\left(106^{\circ}\right.$ and $40^{\circ}$, respectively), which we ascribe to small impurities, metastable equilibrium CA [29], and perhaps the very limited thickness variation in our patterns, we conclude that the increase in $\Theta_{\|}$from the lower limit for clean $\mathrm{SiO}_{2}$ to the upper limit for a PFDTS SAM is adequately described using Cassie's law.

The CAs $\Theta_{\perp}$ perpendicular to the stripes reflect the behavior of the liquid at the chemically defined border between hydrophilic and hydrophobic stripes. At this boundary the droplet is effectively pinned to the border between a PFDTS and a $\mathrm{SiO}_{2}$ stripe. The "last covered stripe" is $\mathrm{SiO}_{2}$. Under equilibrium conditions, the $\mathrm{CA}$ is therefore expected to be close to the equilibrium CA on a PFDTS monolayer. In Fig. 3(c) the dependence of $\Theta_{\perp}$ on the scaling parameter $\alpha$ is shown. As expected, most of the measured $\Theta_{\perp}$ scatter around a value of $104^{\circ}$, slightly less than the equilibrium CA for glycerol on a pristine PFDTS monolayer. Only for the lowest values of $\alpha$ in the range of $0.125-0.25$ is the observed CA well below $100^{\circ}$.

\section{DISCUSSION}

In the following paragraph we attempt to account for our observations by considering the energetics of the surface and discuss the kinetics involved in deposition of the fixedvolume droplets [30]. For advancing (or receding) in the direction parallel to the stripes, the contact line experiences a small constant energy barrier. As a consequence, the contact line of the evolving droplet will advance continuously. In the direction perpendicular to the stripes, the contact line will experience subsequent energy barriers, formed by the hydrophobic PFDTS stripes, giving rise to stick-slip-like motion $[31,32]$.

Initially the evolving droplet exhibits contact angles, exceeding those enforced by the surface chemistry. As such, at first the droplet is expected to spread equally fast in both directions. Preliminary experiments on the kinetics using a high-speed camera indeed confirm that spreading occurs isotropically in the initial stages of equilibration. Moreover, despite the kinetic considerations used in understanding the evolution of the droplet formation, the final shape and therewith the aspect ratio represent an equilibrium situation, which does not depend on the dynamics involved in the movement of the contact line. 
In the direction perpendicular to the stripes, the evolving droplet will be able to overcome the corresponding energy barrier until the energy gain due to minimization of the distortion of the liquid-gas interface (surface tension will always attempt to achieve a spherical droplet shape) will exceed the energy loss caused by spreading over the PFDTS stripes. In other words, the droplet will continue to spread perpendicular to the stripes as long as the actual CA will be greater than the advancing CA for PFDTS $\left(110^{\circ}\right)$. It is worth mentioning that for increasing droplet volumes on a specific pattern, the droplet shape is expected to appear less elongated, i.e., with a lower AR, and eventually approaches a spherical form. Experiments with droplets up to $30 \mu \mathrm{l}$ indeed confirm this.

Parallel to the chemically defined stripes, the droplet (with a fixed volume of $1 \mu \mathrm{l}$ ) will continue to spread along the stripes, until the equilibrium CA $\Theta_{\|}$[in accordance with Cassie's law in Eq. (3)] is reached. For small values $\alpha<1$, the elongation of the droplet required to reach the equilibrium $\Theta_{\|}$is very large. Consequently, due to the constraint that we use droplets of a fixed volume, the equilibrium CA in the direction perpendicular to the stripes will be smaller than the advancing CA on PFDTS and thus advancing of the contact line is inhibited. Accordingly, the observed CAs in Fig. 3 (c) are well below the maximum value of $104^{\circ}$. Moreover, this also accounts for the smaller widths at low $\alpha$ values as shown in Fig. 2(c). In fact, kinetic measurements, which we will report on in a future paper, will reveal the precise behavior.

Finally, with increasing $\alpha$, the values of the $\Theta_{\|}$also increase as shown in Fig. 3(b). The corresponding elongation parallel to the stripes is markedly lower. Due to the con- straint of the fixed volume of our droplets, spreading across the stripes during droplet deposition will continue as long as the actual CA exceeds the advancing CA for PFDTS. This also may well account for the generally observed trend that the width increases with $\alpha$ until the value for the pristine PFDTS SAMs is obtained.

\section{CONCLUSIONS}

Summarizing, we have investigated the three-dimensional equilibrium shape of droplets deposited on anisotropic, chemically heterogeneous surfaces, formed by alternating hydrophilic and hydrophobic stripes with widths in the lowmicrometer range. The experimental results on our chemically patterned surfaces show great resemblance to measurements on morphologically structured surfaces. The aspect ratio of the droplets as well as the contact angles in directions parallel and perpendicular to the stripes exhibit remarkable scaling behavior. As long as the droplet dimensions are 1-2 orders of magnitude larger than the width of the stripes, these quantities do not depend on the absolute size of the surface pattern, but only depend on the relative width of the hydrophobic and hydrophilic stripes.

\section{ACKNOWLEDGMENTS}

The authors thank H. Gardeniers for fruitful discussions and S. de Beer (Physics of Complex Fluids, University of Twente) for AFM verification of the PFDTS self-assembled monolayers. We gratefully acknowledge the support by MicroNed, a consortium that nurtures microsystems technology in The Netherlands.
[1] H. Kusumaatmaja and J. M. Yeomans, Langmuir 23, 6019 (2007).

[2] A. A. Darhuber, S. M. Troian, S. M. Miller, and S. Wagner, J. Appl. Phys. 87, 7768 (2000).

[3] H. Gau, S. Herminghaus, P. Lenz, and R. Lipowsky, Science 283, 46 (1999).

[4] C. Bauer, S. Dietrich, and A. O. Parry, Europhys. Lett. 47, 474 (1999).

[5] C. Bauer and S. Dietrich, Phys. Rev. E 61, 1664 (2000).

[6] M. Brinkmann and R. Lipowsky, J. Appl. Phys. 92, 4296 (2002).

[7] R. Konnur, K. Kargupta, and A. Sharma, Phys. Rev. Lett. 84, 931 (2000).

[8] X.-M. Li, D. N. Reinhoudt, and M. Crego-Calama, Chem. Soc. Rev. 36, 1350 (2007).

[9] L. Gao, A. Y. Fadeev, and T. J. McCarthy, MRS Bull. 33, 747 (2008).

[10] P. Lenz and R. Lipowsky, Phys. Rev. Lett. 80, 1920 (1998).

[11] P. Lenz and R. Lipowsky, Eur. Phys. J. E 1, 249 (2000).

[12] U. Thiele, L. Brusch, M. Bestehorn, and M. Bär, Eur. Phys. J. E 11, 255 (2003)

[13] C. Neinhuis and W. Barthlott, Ann. Bot. (Lond.) 79, 667 (1997).
[14] P. Roach, N. J. Shirtcliffe, and M. I. Newton, Soft Matter 4, 224 (2008).

[15] H. Kusumaatmaja, R. J. Vrancken, C. W. M. Bastiaansen, and J. M. Yeomans, Langmuir 24, 7299 (2008).

[16] W. Li, G. Fang, Y. Li, and G. Qiao, J. Phys. Chem. B 112, 7234 (2008).

[17] R. E. Johnson and R. Dettre, Adv. Chem. Ser. 43, 112 (1964).

[18] D. Xia and S. R. J. Brueck, Nano Lett. 8, 2819 (2008).

[19] J. Y. Chung, J. P. Youngblood, and C. M. Stafford, Soft Matter 3, 1163 (2007).

[20] J. C. Love, L. A. Estroff, J. K. Kriebel, R. G. Nuzzo, and G. M. Whitesides, Chem. Rev. (Washington, D.C.) 105, 1103 (2005).

[21] M. Iwamatsu, J. Colloid Interface Sci. 294, 176 (2006).

[22] P. G. de Gennes, Rev. Mod. Phys. 57, 827 (1985).

[23] M. Morita, T. Koga, H. Otsuka, and A. Takahara, Langmuir 21, 911 (2005).

[24] M. Iwamatsu, J. Colloid Interface Sci. 297, 772 (2006).

[25] Y. V. Naidich, R. P. Vorrovich, and V. V. Zabuga, J. Colloid Interface Sci. 174, 104 (1995).

[26] T. Pompe and S. Herminghaus, Phys. Rev. Lett. 85, 1930 (2000).

[27] A. B. D. Cassie and S. Baxter, Trans. Faraday Soc. 40, 546 (1944). 
[28] M. Gleiche, L. Chi, E. Gedig, and H. Fuchs, ChemPhysChem 2, 187 (2001).

[29] A. Marmur, Soft Matter 2, 12 (2006).

[30] L. Courbin, E. Denieul, E. Dressaire, M. Roper, A. Ajdari, and
H. A. Stone, Nature Mater. 6, 661 (2007).

[31] E. Schäffer and P.-Z. Wong, Phys. Rev. E 61, 5257 (2000).

[32] J. Leopoldes and D. G. Bucknall, J. Phys. Chem. B 109, 8973 (2005). 